\title{
Syndrome de Klinefelter et diagnostic prénatal
}

\author{
R. SAURA
}

Service de Génétique Médicale, Maternité Hôpital Pellegrin, Bordeaux

\section{RESUME}

L'utilisation de plus en plus fréquente du diagnostic prénatal permet de révéler des anomalies gonosomiques, en particulier le syndrome de Klinefelter, non associées à des anomalies échographiques fœtales. Les caractéristiques cliniques et biologiques du syndrome de Klinefelter sont bien connues. Il n'en est pas de même des fonctions intellectuelles dont l'évaluation est essentiellement, déterminée à partir d'études anciennes biaisées. Des études récentes démontrent que les facultés intellectuelles de ces sujets, bien que légèrement abaissées sont proches de la normale. Des études récentes montrent que le principal mode de recrutement des sujets $47, \mathrm{XXY}$, en post natal, est le bilan d'infertilité. La découverte d'un foetus XXY génère toujours une anxiété majeure chez le couple. L'absence de malformation et de retard mental devrait permettre la poursuite de la grossesse. L'annonce de l'anomalie est déterminante. Le taux d'interruption est plus important quand c'est l'obstétricien qui fait cette annonce. Des études récentes et notre étude, bien que peu importante, confirment cette constatation. La tendance actuelle est de ne pas proposer d'interruption médicale pour un "Klinefelter " tout en sachant l'impossibilité de prédire quelle sera la gravité du syndrome.

Mots-clés : Diagnostic prénatal, syndrome de Klinefelter, conseil génétique, interruption médicale de grossesse.

\section{INTRODUCTION}

Le diagnostic prénatal est de plus en plus utilisé dans les pays développés. En France près de 70000 patientes ont un diagnostic prénatal, soit $10 \%$ environ des gestantes. Les indications de diagnostic prénatal des aberrations chromosomiques sont : l'âge maternel, un antécédent d'enfant porteur d'une aberration chromosomique, un parent porteur d'un remaniement chromosomique équilibré, un risque augmenté d'avoir un enfant trisomique par l'étude des facteurs sériques maternels, une anomalie échographique du premier trimestre, en particulier l'augmentation de la clarté nucale ou une anomalie échographique du deuxième ou du troisième trimestre. Ces diagnostics permettent le plus souvent de rassurer le couple en mettant en évidence une formule chromosomique normale, mais dans 2 à $4 \%$ des cas une aberration chromosomique est diagnostiquée. Il peut s'agir d'une anomalie autosomique : trisomie 21,18 ou 13 ou d'autres remaniements de structure déséquilibrés qui sont toujours considérés comme des anomalies sévères. Il peut aussi s'agir de remaniements des chromosomes sexuels qui sont de gravité variable comme le syndrome de Turner, le syndrome de Klinefelter, les triplo $\mathrm{X}$ et les doubles

Correspondance : $R$. Saura, Service de Génétique

Médicale, Maternité Hôpital Pellegrin,

33076 Bordeaux

Communication au XVIIème Congrès de la SALF 7-9 décembre 2000, Bordeaux 
Y. Les conséquences cliniques des anomalies de structure des gonosomes sont variables selon les remaniements impliqués.

La découverte d'une anomalie autosomique déséquilibrée, ou de certains remaniements de structure des gonosomes conduit le corps médical à proposer une interruption médicale de grossesse qui est, à de rares exceptions près, acceptée par le couple. Les anomalies gonosomiques fotales sont d'une gestion bien plus difficile. Une interruption de grossesse est habituellement proposée quand une anomalie échographique est associée à l'anomalie chromosomique, par exemple syndrome de Turner avec un hygroma ou une anasarque. Les autres anomalies gonosomiques : triplo $\mathrm{X}$, syndrome de Klinefelter et les doubles $\mathrm{Y}$ sont des anomalies qui ne génèrent ni malformation majeure ni retard mental et une interruption de grossesse ne devrait, ni être proposée par le corps médical, ni être acceptée si le couple la demande. Mais des études européennes $[9,1]$, et américaines $[16,23,24]$ suggèrent que la plupart des grossesses sont arrêtées. Des publications récentes [11] ont montré que le taux d'interruption de grossesses pour anomalie gonosomique pouvait être diminué.

Les éléments qui vont conduire le couple à prendre une décision sont variables et multiples, ce sont :

- ses convictions personnelles, bien sûr, nous y reviendrons,

- le corps médical et sa propre sensibilité,

- la société et ses lois : " une interruption thérapeutique de grossesse peut être proposée... anomalie d'une certaine gravité... incurable au moment du diagnostic ". Ce qui laisse une grande liberté d'interprétation et une alternative « légale " de continuer ou non la grossesse.

La découverte d'un fotus 47,XXY est une anomalie gonosomique spécifique qui a ses propres caractéristiques. Le " conseiller génétique ", quelle que soit sa formation, doit absolument bien connaître ce syndrome avant de conseiller le couple pour prendre une décision de poursuivre ou non la grossesse.

\section{LE SYNDROME DE KLINEFELTER}

\section{Caractères morphologiques et biolo- giques}

Le syndrome de Klinefelter atteint 1 homme sur 500 quel que soit le groupe ethnique [2], son diagnostic est souvent retardé en raison d'une symptomatologie peu importante et variable. Ces hommes ont une altération importante de la spermatogenèse (habituellement une azoospermie) et différents degrés d'hypotestostéronémie. Durant l'adolescence les anomalies retrouvées sont : une croissance rapide et une obésité troncale. Le risque de cancer du sein est multiplié par 20 [21]. Le taux de testostérone est abaissé, le taux des gonadotrophines est augmenté, la FSH est relativement plus augmentée que la $\mathrm{LH}$. Les formes homogènes : 47, XXY sont habituelles, les formes en mosaïques : $46, \mathrm{XY} / 47, \mathrm{XXY}$ sont plus rares et le diagnostic non fait en raison d'une symptomatologie réduite voire absente. Le traitement repose sur la prise de testostérone, un traitement chirurgical pour la gynécomastie et des ICSI à partir de prélèvements testiculaires ont été rapportées [19] mais le don de spermatozoïdes reste le principal traitement de l'infertilité. Mais le développement post natal des sujets atteints est souvent normal [17].

\section{Fonctions intellectuelles des "Klinefelter ":}

On peut retrouver durant l'adolescence un retard de langage et des difficultés scolaires modérées. Un hypothétique retard mental ou une altération des fonctions intellectuelles seront déterminants dans le choix du couple à poursuivre ou non ces grossesses. Des anomalies du développement des fonctions intellectuelles des sujets atteints de syndrome de Klinefelter ont été rapportées dans la littérature.

La plupart de ces informations concernant les sujets atteints de dysgonosomies ont été obtenues à partir d'études rétrospectives (années 60) faites sur une population recrutée en milieu institutionnalisé ou carcéral et sont donc très biaisées. Elles mettent en évidence 
un nombre élevé de patients porteurs de dysgonosomies et en particulier de syndrome de Klinefelter, avec retard mental (4 à 5 fois plus élevé que celui de la population générale). Ces sujets ne représentent, toutefois, que $1 \%$ des sujets porteurs d'une dysgonosomie. Le QI des enfants atteints de Klinefelter est évalué à 10 à 15 points en dessous des sujets à caryotype normal et ont besoin d'un suivi adapté et peuvent avoir des troubles du comportement [7, 13, 14, 15, 22].

L'évaluation réelle des fonctions intellectuelles des sujets atteints de dysgonosomies a pu être réalisée à partir d'études, non biaisées, sur le suivi à long terme de ces sujets [8]. Des études prospectives récentes [20] ont été réalisées et apportent un éclairage nouveau sur les fonctions intellectuelles des sujets XXY. Cette étude a été réalisée entre 1967 et 1971 et porte sur 72000 nouveau-nés sur lesquels un examen de la chromatine sexuelle a permis de dépister 41 nouveau-nés XXY et 16 nouveaunés XXX. $35 \mathrm{XXY}$ ont un suivi de l'enfance et jusqu'à l'adolescence pour certains.

Un groupe témoin de 32 sujets dont 24 frères ou sœurs des patients étudiés a été suivi dans les mêmes conditions. Des tests de Weschler [25] ont permis d'évaluer certaines caractéristiques intellectuelles des deux groupes. Les tests évaluant le QI, les performances verbales (VIQ) à l'écriture, à la lecture, à l'arithmétique (WRAT) et l'évaluation des performances globales (PIQ) ont été réalisés. Le QI a été de 92,3 pour les XXY et de 103,5 pour les témoins. Les performances verbales ont été respectivement de 84,4 et de 101,3 pour les XXY et pour les témoins. Les performances globales ont été respectivement de 98,7 et de 105,4 pour les XXY et pour les témoins. Bien que ces performances soient légèrement inférieures à celle du groupe témoin, en particulier pour les performances verbales, les sujets XXY ont des performances intellectuelles qui restent dans les limites de la normale.

Dans une autre étude, Robinson et al. [18] montrent que les performances des XXY sont meilleures lorsque le diagnostic a été réalisé en diagnostic prénatal et s'améliorent avec le milieu socio-économique, ce qui n'est pas très surprenant.

\section{CIRCONSTANCES DE DECOUVERTE DES SUJETS 47,XXY}

Le syndrome de klinefelter peut être de découverte fortuite, en diagnostic prénatal ce qui est habituel ou en post natal dans le cadre d'un bilan pour une autre pathologie.

Le diagnostic est parfois évoqué puis confirmé devant :

- des symptomatologies très évocatrices comme une azoospermie, des troubles endocriniens et ou une gynécomastie à l'adolescence chez un garçon.

- d'autres fois il s'agit d'une découverte faite dans le cadre d'un bilan réalisé pour des sujets placés en institutions spécialisées : en psychiatrie ou en milieu carcéral. Le biais de recrutement est évident et afin de mieux évaluer l'incidence et les conséquences réelles du syndrome de Klinefelter, Abramsky et Chapple [1] ont estimé un nombre de rouveau-nés atteints de syndrome de Klinefelter pendant une période de 4 ans (90 à 93) dans une région donnée, et comparé l'estimation aux diagnostics de Klinefelter confirmés par le caryotype en diagnostic pré ou postnatal. Durant cette période 198095 nouveau-nés ont été recensés dont 94547 de sexe masculin. L'estimation de nouveau-nés atteints de syndrome de Klinefelter était de 118 dont 12 ont été diagnostiqués en diagnostic prénatal fortuitement. Sur les 106 restant, $28(26 \%)$ ont été diagnostiqués en post natal. L'âge du diagnostic a été : avant 1 an : 1 cas, entre 11 et 20 ans : 7 cas dont 1 en mosaïque (7\%), après 20 ans : 20 cas dont 1 en mosaïque (19\%). 78 cas de Klinefelter sont donc "passés inaperçus ». Les indications qui ont permis de faire le diagnostic des 28 syndrome de Klinefelter en post natal ont été : 4 retards mentaux (entre 11 et 20 ans), 1 trouble du comportement (après 20 ans), 10 hypogonadismes et ou gynécomasties ( 2 entre 11 et 20 ans et 8 après 20 ans), 5 infertilités (après 20 ans) et 8 indications inconnues ( 1 après 1 an, 1 entre 11 et 20 ans et 6 après 20 ans ). Par ailleurs Abramsky et Chapple [1] ont étudié pendant la même période les indications de caryotype en post natal et extrait parmi les caryotypes anormaux ceux qui avaient une formule $47, \mathrm{XXY}$. 
1594 caryotypes ont été réalisés pendant cette période en post natal. Sur les 1109 qui ont été réalisés pour un retard mental : 1100 étaient 46,XY (dans notre étude personnelle, pour la même indication, nous avons 360 formules normales $46, \mathrm{XX}$ ou $46, \mathrm{XY}) .4$ sujets avaient une formule $47, \mathrm{XXY}(0,36 \%)$ (dans notre étude personnelle; aucun cas $47, \mathrm{XXY}$ et 2 cas $47, \mathrm{XXX}) .5$ sujets avaient une formule $47, \mathrm{XYY}(0,45 \%)$ ( 2 cas dans notre étude personnelle). Un sujet $47, \mathrm{XXY}$ a été retrouvé pour des troubles du comportement. Sur les $\mathbf{3 7 0}$ réalisés pour malformation ; aucun $47, \mathrm{XXY}$ n'a été retrouvé. Sur les 14 caryotypes réalisés pour infertilité ; 4 avaient une formule 47,XXY. Et sur les 91 diagnostics réalisés pour hypogonadisme et ou gynécomasties, 9 avaient une formule $47, \mathrm{XXY}$ et 2 une formule $47, \mathrm{XYY}$.

\section{ETUDE DES SYNDROMES DE KLINEFELTER DU CENTRE DE MEDECINE FOETALE}

Entre 1990 et 1999 nous avons eu 72 patientes dont le fœtus présentait une aneuploïdie gonosomique : $31: 45, \mathrm{X}, 24: \mathrm{XXY}, 11: \mathrm{XXX}$ et 6 XYY. L'âge gestationnel moyen des grossesses était de 14 semaines d'aménorrhées. 13 diagnostics prénataux ont été réalisés dans notre centre dont une grossesse gémellaire monozygote, 10 par choriocentèse et 2 par amniocentèse. 11 patientes ont eu une amniocentèse hors centre et ont été adressées pour un deuxième avis. 4 patientes sur 12 du centre et 4 sur 11 hors centre ont eu une IMG. Le terme moyen de ces IMG a été 15,5 SA.

La découverte d'un fœtus XXY va générer chez le couple une anxiété majeure, qui n'est pas spécifique à cette pathologie, mais qui est la conséquence de l'annonce d'une anomalie, quelle que soit sa gravité, au cours d'une grossesse qui se déroule jusqu'alors normalement. Il est donc nécessaire d'informer le couple le plus objectivement possible, sur les caractéristiques et les conséquences de ce syndrome pour l'enfant à venir. L'annonce d'une infertilité est relativement bien acceptée par le couple. L'aide médicale à la procréation a déjà été proposée à des sujets atteints de syndrome de Klinefelter qui ont pu avoir des enfants nor- maux [19], mais elle ne peut être proposée à tous les sujets XXY. L'absence de dysmorphie et une vie sexuelle normale, pour le sujet XXY sont des informations importantes pour le couple. Il faut toutefois signaler l'impossibilité d'exclure une exceptionnelle forme majeure du syndrome de Klinefelter, ce qui va rendre d'autant plus difficile le conseil génétique.

Peu d'études ont été réalisées à ce jour sur les éléments qui ont conduit les patients à prendre leur décision. Des études européennes $[9,1]$, et américaine $[23,24,16]$ suggèrent que près de $60 \%$ des fœtus XXY sont arrêtés. Dans une publication récente ; Meschede et al. [11] ont montré que le taux d'interruption de grossesses pour anomalie gonosomique pouvait être diminué. Dans leur série, 4 IMG ont été réalisées sur 23 fœtus XXY, ce qui est nettement inférieur aux taux retrouvés dans les autres publications, il est vrai, plus anciennes. En dehors des critères spécifiques au syndrome de Klinefelter déjà vus ; infertilité, syndrome dysmorphique, hypothétique retard mental, d'autres paramètres vont intervenir dans le choix du couple. La découverte d'une anomalie échographique, même si elle est à priori sans rapport avec l'anomalie gonosomique, ce qui est toujours difficile à démontrer, va inciter le couple à demander une interruption médicale de grossesse. Les facteurs culturels et religieux sont aussi des éléments déterminant dans le choix du couple. Le diagnostic prénatal est un acte volontaire, il n'a aucun caractère obligatoire. L'annonce d'une anomalie concernant le foetus, le plus souvent, non prévisible, va générer des réactions, parfois contraires à celles initialement prévues. Le couple est alors en «situation» qu'il doit gérer. Holmes-Siedle et al. [9] ont montré que les IMG étaient d'autant plus demandées que : les patients sont jeunes, que les couples ont peu ou pas d'enfants, que les gens ont effectué des études secondaires ou universitaires. La perception par le couple de l'éventuel handicap, pour l'enfant à venir, joue, très probablement, un rôle important dans le choix de continuer ou non la grossesse. Un autre facteur très déterminant est la qualification du médecin qui réalise l'annonce de l'anomalie et qui réalise le conseil génétique. Il apparaît que le taux d'IMG est diminué lorsque le conseil génétique est réali- 
sé par un généticien plutôt que par un obstétricien [9]. Meschede et al. [11] constatent que le taux d'IMG est plus important quand le couple est adressé au centre pour avoir un deuxième avis. Nous faisons la même observation mais notre série est insuffisante pour le vérifier. Dans notre centre l'annonce est toujours réalisée par le généticien. L'information est directement donnée au couple, au cours d'une consultation réalisée en "urgence ", en évitant, si possible, de donner la nature de l'anomalie afin d'éviter que le couple consulte, entre autre, les données sur internet. L'information, donnée au couple, se doit d'être objective, neutre, non paternaliste, loyale. Le caractère non directif de la consultation de génétique espéré par certains auteurs $[3,5,10$, 12] est tout à fait souhaitable, mais il semble réellement difficile à appliquer du fait des réactions subjectives des protagonistes (couple et médecin) et d'un contexte particulier dû à chaque situation qui est unique.

Dans leur étude Abramsky et Chapple [1] montrent que la plupart des sujets $47, \mathrm{XXY}$, ne sont pas diagnostiqués durant une partie de leur vie. Cela démontre que ces sujets n'ont aucune indication à réaliser un caryotype en raison de l'absence de toute anomalie physique, comportementale ou mentale.

Proposer aux couples qui attendent un enfant $\mathrm{XXY}$, de prendre contact avec des couples qui ont déjà un enfant Klinefelter, dont le diagnostic a été réalisé en diagnostic prénatal, peut rendre service. Mais le recul est insuffisant pour préjuger des éventuelles prédispositions intellectuelles des enfants à long terme. Nous l'avons proposé à plusieurs reprises. Chaque fois le couple a été rasséréné après avoir vu le jeune enfant XXY.

\section{CONCLUSION}

Même en l'absence de consensus la tendance qui se dégage est de proposer de poursuivre la grossesse devant la découverte d'une anomalie gonosomique et en particulier d'un syndrome de Klinefelter. Mais on ne pourra jamais prédire la gravité du syndrome de Klinefelter, ce qui rend parfois le conseil génétique difficile. La mise en place récente des commissions pluris- disciplinaires de diagnostic prénatal peut aider à prendre une décision médicale, mais ne permet pas toujours au couple de retrouver la sérénité d'une grossesse normale et de l'empêcher de verser dans un désarroi parfois irréversible.

\section{REFERENCES}

1. ABRAMSKY L., CHAPPLE J. : 47,XXY (Klinefelter syndrome) and 47,XYY: Estimated rates and indication for postnatal diagnosis with implications for prenatal counselling. Prenatal Diagn., 1997, 17 : 363368.

2. AMORY J.K., ANAWALT B.D., PAULSEN C.A., BREMNER W.J. : Klinefelter's syndrome. Lancet, 2000; $356: 333-335$

3. BARTELS D.M., LEROY B.S., MCCARTHY P., CAPIAN A.L. : Nondirectiveness in genetic counseling: A survey of practitioners. Am. J. Med. Genet., 1997, $72: 172-179$.

4. BENDER B.G., LINDEN M.G., ROBINSON A. : Neuropsychological impairment in 42 adolescents with sex chromosome abnormalities. Am. J. Med. Genet. (Neuropsychiatr. Genet.), 1993, 48 : 169-173.

5. BERNHARDT B.A. : Empirical evidence that genetic counseling is directive: Where do we go from here? Am. J. Hum. Genet., 1997, $60: 17-20$.

6. CLARKE A. : Is non-directive genetic counseling possible? Lancet, 1991, 338 : 998-1001.

7. COHEN F.L., DURHAM. J.D. : Sex chromosome variations in school-age children. J. Sch. Health, $1985,55,99-102$.

8. EVANS J.A., HAMERTON J.L., ROBINSON A. (eds): Children and Young Adults with Sex Chromosome Aneuploidy. 'Birth Defects: 1990, Original Article Series. Vol 26(4). New York: Wiley-Liss.

9. HOLMES-SIEDLE M., RYYNANEN M., LINDENBAUM R.H. : Parental decisions regarding termination of pregnancy following prenatal detection of sex chromosome abnormality. Prenatal Diagn., 1987, 7 : 239-244.

10. KESSLER S. : Psychological aspects of genetic counseling. XI. Nondirectiveness revisited. Am. J. Med. Genet., 1997, 72 : 164-171.

11. MESCHEDE D., LOUWEN F., NIPPERT I., HOLZGREVE W., MINY P., HORST J. : Low rates of pregnancy termination for prenatally diagnosed Klinefelter syndrome and other sex chromosome polysomies. Am. J. Med. Genet., 1998,. 80 : 330-334.

12. MICHIE S., BRON F., BOBROW M., MARTEAU T.M. : Nondirectiveness in genetic counseling : An empirical study. Am. J. Hum. Genet., 1997, 60 : 4047. 
13. NIELSEN J., PELSEN B. : Follow-up 20 years later of $34 \mathrm{~K}$ linefelter males with karyotype $47, \mathrm{XXY}$ and 16 hypogonadal males with karyotype 46,XY. Hum. Genet., 1987, 77 : 188-192.

14. NIELSEN J., WOHLERT M. : Sex chromosome abnormalities found among 34,910 newborn children: results from a 13 year incidence study in Arhus. Denmark, Birth Defects, 1990, 26 : 209-223.

15. RATCLIFFE S.G., BUTLFR G.E., JAMES M. : Edinburgh study of growth and development of children with sex chromosome abnormalities. In : Evans, J.A., Hamerton, J.L., Robinson, A. (eds). Children and Young Adults With Sex Chromosome Aneuploidy, New York : Wiley-Liss for the National Foundation, March of Dimes, 1990 : 59-115.

16. ROBINSON A., BENDER B.G., LINDEN M.G. : Decisions following the intra-uterine diagnosis of sex chromosome aneuploidy. Am. J. Med. Genet., 1989, $34: 552-554$.

17. ROBINSON A., BENDER B.G., UNDEN M.G. : Summary of clinical findings in children and young adults with sex chromosome anomalies. In Evans J.A., Hamerton J.L., Robinson A. eds. Children and Young Adults with Sex Chromosome Aneuploidy. Birth Defects: Original Article Series, Vol 26(4). New York, Wiley-lis, 1990, 225-228.

18. ROBINSON A., BENDER B.G., LINDEN M.G. : Prenatal diagnosis of sex chromosome abnormalities. In Milunsky A. (ed). Genetic Disorders and the Fietus. Diagnosis, Prevention, and Treatment. Baltimore, Johns Hopkins University Press, 1992 : 211-239.

19. RON-EL R., STRASSBURGER D., GELMANKOHAN S., FRIEDLER S., RAZIEL A., APPELMAN Z. : A 47,XXY fetus conceived after ICSI of spermatozoa from a patient with non-mosaic Klinefelter's syndrome. Hum. Reprod., 2000, 15, 1804-1806.

20. ROVET J., NETLEY C., BAILEY J., KEENAN M., STEWART D. : Intelligence and achievement in children with extra $\mathrm{X}$ aneuploidy: A longitudinal perspective. Am. J. Med. Genet. (Neuropsychiatr. Genet.), 1995, 60 : 356-363.

21. SMYTH C.M., BREMNER W.J. : Klinefelter syndrome. Arch. Intern. Med., 1998, 158 : 1309-1314.

22. SORENSEN K. : Physical and mental development of adolescent males with Klinefelter syndrome. Horm. Res., 1992, 37 (Suppl. 3) : 55-61.

23. TANNENBAUM H.L., PERLIS T.E., ARBEITEl, B.E., HSU L.Y.F. : Analysis of decision to continue or terminate pregnancies diagnosed with sex chromosome abnormalities by severity of prognosis, socioeconomic level and sex of fetus. Am. J. Hum. Genet., 1986, 39 (Suppl.) : A183.

24. VERP M.S., BOMBARD A.T., SIMPSON J.L., ELIAS S. : Parental decision following prenatal diagnosis of fetal chromosome abnormality. Am. J. Med. Genet., $1988,29: 613-622$.
25. WECHSLER D. : "Weschler Aduit Intelligence ScaleRevised». New York, The psychological Corporation., 1984.

\section{ABSTRACT}

Antenatal diagnosis of Klinefelter syndrome

$$
\text { R. SAURA }
$$

Because of the widespread use of amniocentesis and chorionic villus sampling, antenatal diagnosis of sex chromosome anomalies, particularly 47,XXY, has become increasingly common. The clinical and laboratory features of Klinefelter syndromes are well documented. On the contrary, the intelligence and achievement outcome of patients with Klinefelter syndrome are less well documented. Early reports suggested an increased frequency of psychological disturbances, subnormal intelligence, and sociopathy; however, because this information was obtained from inmates/patients of various institutions and clinics, it is considered to be biased. Most males born with the $47, \mathrm{XXY}$ pattern will go through life without being karyotyped, while the commonest indication for karyotyping of a Klinefelter male is hypogonadism and/or infertility. Parents are very anxious when they are told that their fetus is $47, \mathrm{XXY}$. The couple should be allowed to continue the pregnancy in view of the absence of developmental delay and/or behavioural problems and congenital malformations. The abortion rate is higher when the fetal abnormality is announced by an obstetrician rather than a geneticist. The current tendency is to no longer propose termination of pregnancy, as most 47,XXY males only present with infertility.

Key-Words: Antenatal diagnosis, Klinefeller syndrome, genetic counselling, termination of pregnancy. 